\title{
Impact of Seasonal Malaria Chemoprevention in Children 5 to 10 Years in Kita and Bafoulabe Districts, Mali.
}

Sory Ibrahim Diawara ( $\sim$ sdiawara@icermali.org )

Malaria Research and Training Center https://orcid.org/0000-0001-5154-2940

Jules Mihigo

USAID

Drissa Konate

Malaria Research and Training Center

Protais Ndabamenye

SAVE THE CHILDREN

Eric Swedberg

Save the Children

Lyndsey W. Garg

Save the Children

Nathalie Gamache

Save the Children

Bourama Keita

Malaria Research and Training Center

Beh Kamate

Save the Children

Philbert Ndaruhutse

Save the Children

Diakalia Kone

NMCP

Vincent Sanogo

NMCP

Moctar Tounkara

Malaria Research and Training Center

Mahamadou Diakite

Malaria Research and Training Center

Seydou Doumbia

Malaria Research and Training Center

Erin Eckert 
USAID

\section{Research}

Keywords: Malaria, children, Kita and Bafoulabe districts, Mali

Posted Date: July 23rd, 2020

DOI: https://doi.org/10.21203/rs.3.rs-36162/v1

License: (c) (1) This work is licensed under a Creative Commons Attribution 4.0 International License. Read Full License 


\section{Abstract}

Background : Seasonal malaria chemoprevention (SMC) is the administration of complete therapeutic courses of antimalarial to all children 3-59 months old during the malaria transmission season. This study measured coverage, impact and cost of adding SMC in children aged 5-10 years.

Methods : A non-randomized, pre-post design, with an intervention (Kita) and control (Bafoulabe) district implemented SMC for children 5-10 years old through the health system in 2017 and 2018. SMC implementation consisted of the administration of SP $+A Q$ at monthly intervals in children 5-10 years in July, August, September and October annually. Baseline and endline household surveys were conducted in both districts. Separate surveys to measure adherence and tolerance to treatment occurred annually in the intervention district ( 200 households) following each of the four treatment rounds. Routine data on malaria cases tested and treated and information on SMC campaign and treatment costs were collected.

Results : A total of 310 and 323 children 5 to 10 years were included in Kita and Bafoulabe respectively in the baseline survey in July 2017, plasmodium infection prevalence was comparable in the two districts ( $p=0.07$ ): $27.7 \%$ in the intervention district (Kita) against $21.7 \%$ in the comparison district (Bafoulabé). Mild anemia was found in $14.2 \%$ of children in Kita vs $10.5 \%$ in Bafoulabé. Household survey found $89.1 \%$ of SMC coverage rate, child's mothers were interviewed with $93.3 \%$ during the SMC campaign in Kita. The most side effect reported by parents was vomiting with $9.3 \%$. One year after SMC implementation in 5 to 10 years in Kita, three doses coverage was $81.2 \%$, there was a reduction by $40 \%$ $(\mathrm{OR}=0.60, \mathrm{Cl}: 0.41-0.89)$ of malaria parasite carriage; $21 \%$ and $62 \%$ reduction of simple malaria and severe malaria prevalence respectively in the pilot district vs control. Mild anemia and severe anemia were comparable in the two districts. The level of malaria molecular resistance rate remains below the threshold. Quintuple mutation (dhfr triple +dhps437+dhps540) remained $<5 \%$ after intervention in both districts.

Conclusion: The SMC strategy contributed to malaria prevention in 5-10 year old children and should be integrated to SMC for children 3-59 months. Keywords : Malaria, Seasonal Malaria Chemoprevention, Sulfadoxine-Pyriméthamine, Amodiaquine, Anemia.

\section{Background}

Malaria remains a major cause of morbidity in the African Sahel sub-region, with clinical cases estimated at 228 million with 405,000 deaths in 2018 (1). Regular administration of recommended antimalarial drugs during the rainy season is an effective means of preventing malaria morbidity and mortality in children (2). In 2012, WHO recommended Seasonal Malaria Chemoprevention (SMC) for malaria prevention in Sub-Saharan African countries with highly seasonal malaria transmission and an absence of SP resistance, including Mali, where the malaria prevalence in children under 5 years of age is $19 \%$ (DHS 2018). Routine surveillance data shows an almost three times higher prevalence of cases in older children: $58.1 \%$ versus $19 \%$ in 3 months to 5 years. Among children 5 to 14 years of age, mortality due to 
malaria is $4 \%$ in the Kayes region (SLIS 2016). Essential interventions currently recommended by WHO, seasonal malaria chemoprevention (SMC) for children under 5 years provides substantial protection as shown by studies in the sub-region (3-6). Following promising findings of these studies, WHO recommended SMC in areas where malaria burden in the first years of life is high and SulfadoxinePyrimethamine resistance has not yet developed. (7). In Senegal, the neighboring country, JL Ndiaye et al found a substantial benefit of SMC among children 5-9 years of age, on hemoglobin and the prevalence of parasitemia (8). Several studies have shown the epidemiological impact of SMC in school age children in clinical trials (8-10). Touré et al in 2016 found that the proportion of children over 5 years of age carrying malaria parasites was higher in all seasons compared to younger children in Sélingué, Mali due to malaria control policy focused on children under five and pregnant women. (11). Elsewhere, in African school-age children, malaria prevalence and incidence have increased as shown by Nankabirwa et al in 2014 (12). These children use Long-lasting insecticide treated bed nets (LLINs) less frequently than other population groups, may not have access to free anti-malarial treatment, and are not protected by routine SMC to control malaria. The focus on children under 5 years may cause a delay in the acquisition of natural immunity, causing older children to be at increased risk of acute infection once they have outgrown the age-specific control interventions targeted to under 5 years old children as showed by Pemberton-Ross, $\mathrm{P}$ and al; Ndiaye, $\mathrm{M}$ and al $(13,14)$ In the past, school-age children have not received adequate attention as a group at risk for malaria. Additional measures to protect them against malaria are warranted, including SMC extension and improved use of LLINs. .The current study aimed to assess the effect of seasonal malaria chemoprevention on anemia and malaria parasitemia among children 510 years in Kita district.

\section{Methods}

\section{Study Site}

The study was undertaken in two neighboring health districts (Kita and Bafoulabe) in the health district of Kita, located in western Mali. The two districts have a combined population of about 670,000 (RGPH $2008,(15))$ that are served by 67 community health centers and two referral health centers (equivalent to a district hospital). The study areas have the same geographic, demographic and epidemiological characteristics including a sahelian climate with a single rainy season from July to October. SMC implementation covering children 3-59 months has been occurring in the district of Kita by the National Malaria Control Program since 2014, with four monthly rounds from August to November for two years (2014, 2015, and 2016).

\section{Study Design}

This non-randomized, pre-post design study was conducted among children aged 5 to 10 years in both an intervention (Kita) and control (Bafoulabe) district. 
Sample size: A recent Cochrane review on SMC, estimated that SMC can reduce parasitemia by $65 \%$. For this study, we assume a $50 \%$ reduction under programmatic conditions. To detect a $50 \%$ difference in parasitemia in older age group between the normal SMC and extended SMC districts at follow-up, with alpha $=0.05$, power of $80 \%$ and assuming $12.5 \%$ and $25 \%$ parasitemia in the district at follow-up respectively and design effect of 2.0,304 children aged 5-10 years would be needed to be surveyed in each district. Assuming one child 5-10 years old per household, survey teams would need to visit 304 households in each of the two districts at baseline and follow-up each year (total sample size $=608$ household at baseline and 304 at follow-up for the 2 years of data collection; 1,216 children from 5 to 10 years were assessed). In each district, 304 households were randomly selected according to adherence to the study after signed consent.

At baseline 323 children in Bafoulabé and 310 in Kita were enrolled in the study. For post-intervention surveys, a total of 279 children in Bafoulabé district and 372 children in Kita district were enrolled in the study. One child by household was randomly selected in the selected village. SMC implementation consisted of the administration of SP + AQ at monthly intervals in children aged 3-59 months old and to children aged 5 to 10 years in July, August, September and October 2017 and 2018 to all eligible children.

To estimate parasitic infection and anemia, we conducted a cross-sectional survey in the two districts one month after the 4th round of SMC.

Written informed consent was obtained from all participants' mothers or guardians prior to inclusion. Each child gave a blood sample for hemoglobin measurement using a HemoCue device, and for malaria parasite detection using a blood smear and filter paper for molecular markers of the resistance to SP and $\mathrm{AQ}$

Drug adverse events were monitored by household visit 4-7 days after each of the four rounds of SMC in the intervention district of Kita. Community based distributors (CBD) checked any reactions to treatment, documented and reported to the nearest health center. Laboratory investigation were conducted in cases of severe skin reactions and signs of liver disease.

\section{Delivery and Drugs}

A therapeutic dose of the standard drug regimen per WHO, AQ (10 mg/kg/day for 3 days) combined with one dose of SP on the first day ( $25 \mathrm{mg}$ Sulfadoxine and $1.25 \mathrm{mg}$ pyriméthamine per $\mathrm{kg}$ ) was administered once per month from July to October to all children 3-59 months old in both districts and to children from 5 to 10 years in Kita; SMC first dose was given by CBDs by door to door strategy and the subsequent doses were given by mothers or the child's guardian. For data monitoring of the first dose $n$ was recorded in registers maintained by the CBDs. The administration at home was recorded to SMC cards maintained by the parents. 
HemoCue device (Angelholm, Sweden) was used to measure hemoglobin levels in the field among children who had blood samples by finger prick.

Anemia classification according DeMaeyer was used (16):

Children with hemoglobin level below $8.0 \mathrm{~g} / \mathrm{dl}$ were classified as patients with severe anemia; between 8.0 and $9.9 \mathrm{~g} / \mathrm{dL}$ for moderate anemia and between 10.0 and $10.9 \mathrm{~g} / \mathrm{dl}$ for mild anemia.

The thick and thin smears were performed in each child participating in the study. The slides were labeled with a unique identification number of child and dated. The slides were dried in open air in slide boxes and then transferred at the end of day in slide boxes for safe storage. Thin smears were fixed with methanol. The slides were stained using Giemsa $10 \%$ and transported to the laboratory in the Malaria Research and Training Center in which they were read by two independent readers.

Malaria disease was defined as axillary temperature $\geq 37.5^{\circ} \mathrm{C}$ and the presence of asexual malaria parasites in the blood smear. In addition to previous conditions, prostration or convulsions or coma will be considered as severe malaria and requested specific management.

\section{Surveillance}

Confirmed malaria data from July to December 2017 and July to December 2018 were collected from outpatient registers in community health centers. A total of 5 centers were randomly selected in each of the two districts from the ten best performing centers in each district based on certain performance criteria (accuracy, completeness of consultation registers). This passive surveillance allowed for the assessment of trends of malaria burden in children aged 5-10 years.

\section{Data management and analysis}

Data on each participant were collected by the study team on the Case Report Forms (CRF) kept in place in accordance with good clinical practices. These data from household survey and health facilities were double entered in ACCESS 2010 by two different operators. The prevalence of anemia and malaria parasitemia was compared in intervention and control districts one month after the fourth round of SMC. Logistic regression was used for binary outcomes of anemia, malaria disease and parasitemia, and used a difference-in-differences approach. The level of significance was set at $5 \%$.

\section{Assessment of molecular markers of resistance to SP and $\mathrm{AQ}$}

Molecular analysis was performed on samples from children with hose blood smear showed $P$. falciparum parasitaemia $\geq 160 /$ uL (4 parasites/200 WBC) by blood smear. Blood samples on paper filter 
were analysed by nested polymerase chain reaction (PCR) and/or PCR-restriction fragment length polymorphism (RFLP) for mutations at codons 51, 59 and 108 of the dhfr gene, 437 and 540 of the dhps gene, mutations at codon 76 in the $P$. falciparum chloroquine transporter gene (pfcrt), and at codon 86 of the $P$. falciparum multidrug resistance gene one (pfmdr1) according to published methods $(17,18)$. Cases of mixed infection (wild type and mutant) were categorized as mutant. Quintuple mutant was defined as the presence of the three dhfr mutations (N51I, C59R and S108N) and the two dhps mutations (A437G and K540E).

\section{Ethical Considerations}

The study protocol was approved by the ethics committees of Faculty of Medicine and Odontostomatology of Bamako and CDC in Atlanta. Written informed consent from parents/ guardians was obtained for all participants by explaining the aims and activities prior to seeking signed consent. The study objectives and data collection procedures were explained in detail to administrative, community and health authorities. Only ID numbers of participants were on the samples and CRFs to ensure anonymity and confidentiality.

\section{Results}

\section{Coverage and Adherence}

In Kita's household survey, 322 and 340 children aged 5 to 10 years were included in 2017 and 2018 respectively. SMC coverage of 3 days treatment for all four rounds according to parent/guardian report was $89.1 \%$ and $81.2 \%$ in 2017 and 2018 , respectively (Fig. 1).

Gender distribution was similar in the two districts for both surveys. At baseline, $43.9 \%$ of children in Kita and $46.4 \%$ in Bafoulabe were male, $(p=0.51)$. These proportions in post-intervention survey were $50.5 \%$ and $45.2 \%$, respectively, in Kita and Bafoulabe $(p=0.17)$.

\section{Parasitemia and Anemia Prevalence}

At baseline, parasite prevalence was similar in the two districts $(23.3 \%$ in Kita vs $27.1 \%$ in Bafoulabe; $p=$ 0.28) (Table 2). In post intervention period, the parasite prevalence was $22.0 \%$ in Bafoulabe district compared to $16.9 \%$ in the intervention district (Kita). A reduction of $40 \%$ in parasite prevalence was observed in the intervention district $(\mathrm{OR}=0.60,95 \% \mathrm{Cl} 0.41-0.89, \mathrm{p}<0.001)$. Fever (defined as an axillary temperature $\left.\geq 37.5^{\circ} \mathrm{C}\right)$ prevalence was similar across the two districts at baseline $(8.0 \%$ vs $8.6 \% ; \mathrm{p}=$ $0.76)$ and higher in the intervention district in post-intervention $(21.2 \%$ vs $11.5 \% ; p=0.001)$ (Table 2$)$. Malaria disease was also similar in the two districts at baseline $(4.8 \%$ vs $5.1 \%, p=0.85)$ after intervention malaria disease prevalence was $13.1 \%$ and $4.3 \%$ respectively in Kita and Bafoulabe $\left(p=10^{-4}\right)$. The prevalence of anemia was similar before and after intervention in the two districts $(13.0 \%$ vs $11.8 \%, p=$ 
$0.64 ; 20.2 \%$ vs $17.2 \% ; p=0.34$ ) respectively (Table.2). The difference in-differences regression models was used to assess and compare changes between the intervention and comparison districts, baseline assessment did not affect the outcome of the final evaluation of parasitemia, malaria disease and anemia as shown in Table.3.

\section{Routine Cases of Malaria}

According to routine data among children from 5 to 10 years from health centers, prior to SMC implementation, the prevalence of confirmed malaria was similar in the two districts $(8.9 \%$ vs $9.4 \% ; p=$ 0.75). After implementation there was a reduction of $21 \%$ in prevalence of simple malaria ( $O R=0.79 ; 95 \%$

$\left.\mathrm{Cl} 0.72-0.88 ; \mathrm{p}<10^{-4}\right)$ in Kita. The same trend was observed for severe malaria with $62 \%$ reduction in the intervention district $\left(\mathrm{OR}=0.38 ; 95 \% \mathrm{Cl} 0.33-0.44 \mathrm{p}<10^{-4}\right)$ (Fig. 2).

\section{Resistance to SMC Drugs}

The prevalence of quadruple mutants (dhfrtriple + dhps437) and quintuple mutants (dhfr triple + dhps 437 + dhps 540 ) remained low ( $<50 \%$ and $<5 \%$ respectively) after intervention in both districts (Fig. 3 ).

\section{Adverse Events}

Regarding reported side effects occurring in 2017 , drowsiness was frequently reported in $11.8 \%$ of children, followed by vomiting in $9.1 \%$, diarrhea in $8.8 \%$ and itching was reported in $2.1 \%$ (Table.1).

\section{Costs}

Considering SMC campaign for children 5-10 years old, the cost per child for the 4 rounds was estimated at US\$3.56. The costs include: supplies, drugs, logistic, orientation/training of SMC team, supervisors, transportation, supervision and community mobilization and restitution workshops. The additional cost for extending SMC to 5-10 years was US\$0.30 per covered child with four rounds. About $86 \%$ of the additional costs are related to drug provision in school age children.

\section{Discussion}

This effectiveness study in Malian school-aged children shows the potential of SMC to reduce parasitemia and both uncomplicated and severe malaria, but no apparent effect on anemia similar to Thera and al study of SMC using artesunate - amodiaquine in school aged children in Mali.(19). The parasite prevalence was similar in intervention and control districts before SMC implementation (23.3\% vs $27.1 \%, p=0.28)$. SMC intervention among children aged from 5 to 10 years was associated with a $40 \%$ reduction of parasite prevalence (16.9\% in Kita vs $22.0 \%$ in Bafoulabé). Anemia prevalence in children 5 
to 10 years was similar prior $(13.0 \%$ vs $11.8 \%, p=0.64)$ and after SMC implementation $(20.2 \%$ vs $17.2 \%$, $p=0.34$ ) respectively in intervention and comparison districts. The prevalence of uncomplicated malaria and severe malaria was reduced by $21 \%$ and $62 \%$ respectively in the intervention district of Kita compared to Bafoulabe (control). Malaria intervention improvement and the high coverage of SMC among children $(>80 \%$ ) could contribute to reflect these findings and is similar to results of other studies, except for the effect on anemia.

During SMC implementation, reported side effects were drowsiness (11.8\%), vomiting (9.1\%) and diarrhea (8.8\%). In previous studies (Dicko \& al in 2011 in Mali (20); Bojang \& al in 2010 in Gambia (21), frequency of side effects is similar to that reported in this study, but had reported less frequent reported side effects with $4 \%$ for diarrhea and $6.7 \%$ for vomiting. However, Bojang \& al found $15.2 \%$ for diarrhea and $15.4 \%$ for vomiting. These results were observed among children less than five years old. These differences could be due to health workers administering the dose on day 1 , versus mothers on days 2 and 3 . Also school age children can express their opinion about medicine taken during SMC period and promptly report any reaction. These side effects were resolved spontaneously without any other medication. In Senegal, Ndiaye, J. A. et al (22) found vomiting as the most frequently reported adverse event in children under 10 years of age who have received SMC treatment and attributed this reaction to amodiaquine.

Our study shows no increase in the frequency of molecular markers of SP and $\mathrm{AQ}$ resistance in SMC areas with SP/AQ was implemented. The frequency of quadruple mutants and quintuple mutants was still low ( $<50 \%$ and $<5 \%$ respectively) after the fourth year of SMC implementation. Based on these results, SP maintains it's efficacy in SMC as shown by other authors (23-26).

Children enrolled in baseline survey in July 2017 were not all found in final survey in December 2018. Preand post-SMC assessments (July 2017 and November 2018) were carried out at different times from the malaria transmission. In the national HMIS system-, age groups did not correspond to those of our study ( $<5$ years and $\geq 5$ years vs $3-59$ months and 5 to 10 years) which limited the use of this database for comparison.

\section{Conclusion}

This study demonstrates the achievement of hight coverage of SMC coverage and adherence in the intervention district and significant reductions of malaria parasitemia and uncomplicated and severe malaria prevalence in 5-10 year old children in the intervention district. The drugs are well tolerated and the prevalence of molecular markers associated with resistance to SP and AQ is still low. The mininal additional costs of adding children 5-10 years old to the SMC intervention are low and are largely due to the medication costs.

\section{List Of Abbreviations}

AQ: Amodiaquine 
CBD: Community based distributors

CHW: Community Health Workers

DHIS2: District Health Information System 2

DHS: Demographic Health Survey

EPI: Expended Program on Immunization

HMIS: Health information management system

IPTi: Intermittent Preventive treatment in Infant

ITN: Insecticide Treat Net

LLIN: Long-lasting Insecticide Treated bed Net

SMC: Seasonal Malaria Chemoprevention

SP: Sulfadoxine-Pyriméthamine

WHO: World Health Organization

\section{Declarations}

\section{Ethics approval and consent to participate}

Ethical clearance for this study was provided by the Ethics Committee of the Faculty of Medicine, Pharmacy, and Odontostomatology, University of Bamako (N²017/67/CE/FMPOS). Written informed consent was obtained from parents or guardians of participants aged from 5 to 10 years.

\section{Consent for publication}

Yes

\section{Competing interests}

The authors declare that they have no competing interests.

\section{Funding}


This research was funded by the United States Agency for International Development (USAID) project Services de Sante a Grand Impact (SSGI) in Mali through the President's Malaria Initiative.

\section{Authors' contributions}

EE, DSI, JM designed the study. KD, TM, BK, OD, TM, ES, CS, DSI and KD coordinated and carried out field activities. KD, DSI, TM performed data analyses. DSI, KD, NP, DM, DS prepared initial draft of the manuscript; NG, NP, EE and LS performed critical review and revision of the manuscript. All authors read and approved the final version of the manuscript.

\section{Acknowledgements}

The authors are very grateful to the study participants, community health centers and the staff of the health Districts of Kita and Bafoulabe for their cooperation throughout the study.

We are grateful to the USAID/SSGI team and to the research team and National Malaria Control Program

\section{References}

1. WHO. World Malaria Report 2019. Geneva. 2019.

2. WHO. World Malaria Report 2015. Geneva. 2015.

3. Cisse B, Sokhna C, Boulanger D, Milet J, Ba el H, Richardson K, et al. Seasonal intermittent preventive treatment with artesunate and sulfadoxine-pyrimethamine for prevention of malaria in Senegalese children: a randomised, placebo-controlled, double-blind trial. Lancet. 2006;367(9511):659-67.

4. Kweku M, Liu D, Adjuik M, Binka F, Seidu M, Greenwood B, et al. Seasonal intermittent preventive treatment for the prevention of anaemia and malaria in Ghanaian children: a randomized, placebo controlled trial. PLoS One. 2008;3(12):e4000.

5. Bojang KA, Akor F, Conteh L, Webb E, Bittaye O, Conway DJ, et al. Two strategies for the delivery of IPTc in an area of seasonal malaria transmission in the Gambia: a randomised controlled trial. PLoS Med. 2011;8(2):e1000409.

6. Konate AT, Yaro JB, Ouedraogo AZ, Diarra A, Gansane A, Soulama I, et al. Intermittent preventive treatment of malaria provides substantial protection against malaria in children already protected by an insecticide-treated bednet in Burkina Faso: a randomised, double-blind, placebo-controlled trial. PLoS Med. 2011;8(2):e1000408.

7. World Health Organization. WHO Policy recommendation on Intermittent Preventive Treatment during infancy with sulphadoxine-pyrimethamine (SP-IPTi) for Plasmodium falciparum malaria control in Africa. Geneva; 2010.

8. Ndiaye JLA, Ndiaye Y, Ba MS, Faye B, Ndiaye M, Seck A, et al. Seasonal malaria chemoprevention combined with community case management of malaria in children under 10 years of age, over 5 
months, in south-east Senegal: A cluster-randomised trial. PLoS Med. 2019;16(3):e1002762.

9. Nankabirwa JI, Wandera B, Amuge P, Kiwanuka N, Dorsey G, Rosenthal PJ, et al. Impact of intermittent preventive treatment with dihydroartemisinin-piperaquine on malaria in Ugandan schoolchildren: a randomized, placebo-controlled trial. Clin Infect Dis. 2014;58(10):1404-12.

10. Barger B, Maiga $\mathrm{H}$, Traore $\mathrm{OB}$, Tekete $\mathrm{M}$, Tembine I, Dara A, et al. Intermittent preventive treatment using artemisinin-based combination therapy reduces malaria morbidity among school-aged children in Mali. Trop Med Int Health. 2009;14(7):784-91.

11. Toure M, Sanogo D, Dembele S, Diawara SI, Oppfeldt K, Schioler KL, et al. Seasonality and shift in age-specific malaria prevalence and incidence in Binko and Carriere villages close to the lake in Selingue, Mali. Malar J. 2016;15:219.

12. Nankabirwa J, Brooker SJ, Clarke SE, Fernando D, Gitonga CW, Schellenberg D, et al. Malaria in school-age children in Africa: an increasingly important challenge. Trop Med Int Health. 2014;19(11):1294-309.

13. Pemberton-Ross P, Smith TA, Hodel EM, Kay K, Penny MA. Age-shifting in malaria incidence as a result of induced immunological deficit: a simulation study. Malar J. 2015;14:287.

14. Ndiaye M, Sylla K, Sow D, Tine R, Faye B, Ndiaye JL, et al. Potential Impact of Seasonal Malaria Chemoprevention on the Acquisition of Antibodies Against Glutamate-Rich Protein and Apical Membrane Antigen 1 in Children Living in Southern Senegal. Am J Trop Med Hyg. 2015;93(4):798800 .

15. RGPH. Recensement Général de la Population et de l'Habitat. 2009.

16. DeMaeyer EMPDea. Preventing and controlling iron deficiency anemia through primary health care, A.g.f.h.a.a.p. managers. Organisation Mondiale de la Santé : Genève. 1989.

17. Plowe CV, Djimde A, Bouare M, Doumbo O, Wellems TE. Pyrimethamine and proguanil resistanceconferring mutations in Plasmodium falciparum dihydrofolate reductase: polymerase chain reaction methods for surveillance in Africa. Am J Trop Med Hyg. 1995;52(6):565-8.

18. Djimde A, Doumbo OK, Cortese JF, Kayentao K, Doumbo S, Diourte Y, et al. A molecular marker for chloroquine-resistant falciparum malaria. N Engl J Med. 2001;344(4):257-63.

19. Thera MA, Kone AK, Tangara B, Diarra E, Niare S, Dembele A, et al. School-aged children based seasonal malaria chemoprevention using artesunate-amodiaquine in Mali. Parasite Epidemiol Control. 2018;3(2):96-105.

20. Dicko A, Diallo Al, Tembine I, Dicko Y, Dara N, Sidibe Y, et al. Intermittent preventive treatment of malaria provides substantial protection against malaria in children already protected by an insecticide-treated bednet in Mali: a randomised, double-blind, placebo-controlled trial. PLoS Med. 2011;8(2):e1000407.

21. Bojang K, Akor F, Bittaye O, Conway D, Bottomley C, Milligan P, et al. A randomised trial to compare the safety, tolerability and efficacy of three drug combinations for intermittent preventive treatment in children. PLoS One. 2010;5(6):e11225. 
22. Ndiaye JA, Diallo I, Y ND, Kouevidjin E, Aw I, Tairou F, et al. Evaluation of Two Strategies for Community-Based Safety Monitoring during Seasonal Malaria Chemoprevention Campaigns in Senegal, Compared with the National Spontaneous Reporting System. Pharmaceut Med. 2018;32(3):189-200.

23. Grais RF, Laminou IM, Woi-Messe L, Makarimi R, Bouriema SH, Langendorf C, et al. Molecular markers of resistance to amodiaquine plus sulfadoxine-pyrimethamine in an area with seasonal malaria chemoprevention in south central Niger. Malar J. 2018;17(1):98.

24. WHO. WHO Policy recommendation on Intermittent Preventive Treatment during infancy with sulphadoxine-pyrimethamine (SP-IPTi) for Plasmodium falciparum malaria control in Africa. Geneva. 2010.

25. Dicko A, Sagara I, Djimde AA, Toure SO, Traore M, Dama S, et al. Molecular markers of resistance to sulphadoxine-pyrimethamine one year after implementation of intermittent preventive treatment of malaria in infants in Mali. Malar J. 2010;9:9.

26. Lo AC, Faye B, Ba el H, Cisse B, Tine R, Abiola A, et al. Prevalence of molecular markers of drug resistance in an area of seasonal malaria chemoprevention in children in Senegal. Malar J. 2013;12:137.

\section{Supplementary Files}

This is a list of supplementary files associated with this preprint. Click to download.

- 12TitlePage.docx 